\title{
Variabilidade espacial da porosidade total, umidade e resistência do solo à penetração de um Argissolo amarelo
}

\section{Spatial variability of total porosity, moisture and soil resistance to penetration of a yellow ultisol}

\author{
Renildo Luiz Mion ${ }^{1 *}$; Elivânia Maria Sousa Nascimento²; \\ Francisco Alexandre de Lima Sales ${ }^{3}$; Silvia Ferreira da Silva ${ }^{4}$; \\ José Moacir Lima Duarte ${ }^{4}$; Beatriz Marques de Sousa ${ }^{3}$
}

Resumo

O estudo da variabilidade espacial de atributos do solo é importante em áreas sob diferentes usos e manejos. A umidade e a resistência do solo à penetração são consideradas um parâmetro indicativo da qualidade física do solo. Objetivou-se com este trabalho estudar a variabilidade espacial da porosidade total, resistência do solo à penetração e da umidade em uma área de pastejo rotacionado de ovinos. $\mathrm{O}$ experimento foi conduzido em um Argissolo Amarelo de textura arenosa nas camadas de 0-0,1, 0,1-0,2 e $0,2-0,3 \mathrm{~m}$. Para a determinação dos atributos e resistência do solo à penetração foi delimitada uma malha, com intervalos regulares de $30 \mathrm{~m}$, perfazendo um total de 13 pontos. A porosidade total (PT) e a umidade gravimétrica foram obtidas através das coletas de amostras indeformadas (amostrador do tipo Uhland) e deformadas, respectivamente. A resistência do solo à penetração (RP) foi determinada em cada ponto, utilizando um penetrômetro eletrônico georreferenciado. Os resultados mostraram que a PT apresentou um coeficiente de variação baixo em todas as camadas estudadas. A US em todas as camadas avaliadas e a RP nas camadas de $0-0,1$ e $0,1-0,2$ m apresentaram um coeficiente de variação médio. A RP na camada de $0,2-0,3 \mathrm{~m}$ apresentou um coeficiente de variação alto mostrando uma distribuição com alta heterogeneidade nos dados. Os atributos PT, RP e US apresentaram um índice de dependência espacial (IDE) fraco em todas as camadas avaliadas. A RP aumenta à medida que a PT e a US exerce uma menor influência sobre o solo.

Palavras-chave: Índice de cone, geoestatística, penetrógrafo

\begin{abstract}
The study of spatial variability of soil attributes is important in areas under different uses and management. The moisture and soil resistance to penetration are considered an indicative parameter of soil physical quality. The objective of this work to study the spatial variability of total porosity, soil resistance to penetration and moisture in an area of rotational grazing sheep. The experiment was conducted in a Yellow Ultisol with a sandy texture in the layers of 0- 0.1, 0.1-0.2 and 0.2-0.3 m. For the determination of properties and soil resistance to penetration was defined a grid, with regular intervals
\end{abstract}

\footnotetext{
${ }^{1}$ Eng $^{\mathrm{o}}$ Agrícola, Prof. Adjunto, Instituto de Ciências Agrárias e Tecnológicas, Universidade Federal do Mato Grosso, UFMT, Campus Universitário de Rondonópolis. E-mail: renildomion@gmail.com

${ }^{2}$ Eng $^{\mathrm{a}}$ Agr $^{\mathrm{a}}$, Mestranda em Engenharia Agrícola, Universidade Federal do Ceará, UFC, Dept ${ }^{\circ}$ de Engenharia Agrícola, Bloco 804, Campus do Pici, Fortaleza, CE. CEP: 60455-760. E-mail: elivania_sousa@yahoo.com.br

${ }^{3}$ Tecnólogos em Irrigação, Mestrando(a) em Engenharia Agrícola, UFC, Dept ${ }^{\circ}$ de Engenharia Agrícola, Fortaleza, CE. E-mail: alexandre@yahoo.com.br; marquessousa@yahoo.com.br

${ }^{4}$ Engenheiros Agrônomos, Mestrando(a) em Engenharia Agrícola, UFC, Dept ${ }^{\mathrm{o}}$ de Engenharia Agrícola, Fortaleza, CE. E-mail: silviasilva@yahoo.com.br; duartelima@yahoo.com.br

* Autor para correspondência
} 
of $30 \mathrm{~m}$, total of 13 points. The total porosity (TP) and the gravimetric soil moisture (SU) were obtained by collecting undisturbed samples (Uhland soil sampler) and disturbed samples, respectively. The soil resistance to penetration (PR) was determined at each point using an electronic georeferenced penetrometer. The results showed that TP had a low variation coefficient in all studied layers. The SU in all evaluated layers and the PR in layers $0-0.1$ and $0.1-0.2 \mathrm{~m}$ showed a medium variation coefficient. The PR in layer 0.2-0.3 m showed a high variation coefficient showing the average distribution with a high heterogeneity in the data. The attributes TP, PR and SU showed a weak spatial dependency index (SDI) in all evaluated layers. The PR increases as the TP and the SU has a smaller influence on the soil.

Key words: Cone index, geostatistic, penetrometer

\section{Introdução}

O estudo da variabilidade espacial do solo é particularmente importante em áreas onde o mesmo está submetido a diferentes manejos (SOUZA et al., 2001), pois a análise geoestatistica pode indicar alternativas de manejo não só para reduzir os efeitos da variabilidade do solo sobre a produtividade agrícola (TRANGMAR et al., 1985) mas também para aumentar a possibilidade de se estimarem respostas dos atributos do solo em função de determinadas práticas de manejo (OVALLES; REY, 1994).

Opotencial produtivodas pastagensé determinado por diversos fatores que estão relacionados ao clima, ao solo e á espécie envolvida, dentre eles, o solo pode impor limitações ao crescimento das plantas pela capacidade de suprimento com nutrientes, água e oxigênio (IMHOFF; SILVA; TORMENA, 2000).

Segundo Carvalho et al. (2007), a umidade do solo pode ser utilizado com indicador do tipo e qualidade do solo. A umidade nas camadas do solo apresenta uma variabilidade importante nos domínios espacial e temporal, que pode levar a manejos inadequados da água do solo (HU et al., 2008). De acordo com Timm et al. (2006), a umidade exerce uma influência sobre importantes processos no solo e na planta tais como: movimento de água, compactação do solo, aeração do solo e desenvolvimento radicular.

Outro parâmetro considerado que indica a qualidade física do solo é a resistência do solo à penetração onde é possível identificar zonas compactadas e auxiliar na escolha do método apropriado para descompactação do solo, facilitando assim o crescimento e o desenvolvimento radicular das plantas (CUNHA; VIEIRA; MAGALHÃES, 2002).
A resistência do solo à penetração é uma das propriedades físicas que expressa o grau de compactação, e consequentemente a facilidade com que as raízes penetram no solo (FUENTES LLANILLO et al., 2006). A avaliação da compactação do solo é baseada na condição atual em que se encontra o solo, sendo possível identificar as camadas que apresentam restrições a fenomenos de superfície, crescimento e produtividade das culturas (SILVA; REICHERT; REINERT, 2004).

A resistência que um solo oferece à penetração é realizada através de um instrumento de sondagem conhecido como penetrômetro o qual reflete o comportamento do solo quanto à compactação, conteúdo de água, granulometria e tipo de argila e dependente de atributos do solo como: textura, porosidade, estrutura, estabilidade de agregados e teor de água (CAMARGO; ALLEONI, 1997; SILVA; REINERT; REICHERT, 2000). Os solos agrícolas podem ser compactados por animais em pastejo, bem como pelas raízes de árvores, porém mais significativos são os efeitos da mecanização, ocasionados pelo tráfego de máquinas Ugadas no manejo das culturas (CUNHA; VIEIRA; MAGALHÃES, 2002).

O solo é um sistema trifásico disperso, a caracterização de sua porosidade total é de grande importância para adoção de um manejo adequado, pois este sistema está estreitamente ligado à dinâmica do armazenamento e do movimento de solutos e de circulação de gases no seu interior, essenciais aos processos bioquímicos das plantas, sobretudo aqueles relacionados com a produtividade vegetal (KIEHL, 1979; EPSTEIN; BLOOM, 2006).

A análise geoestatística constitui uma importante ferramenta na análise e descrição da variabilidade 
das propriedades do solo (CARVALHO; SORATTO; FREDDI, 2002), alem disso o Ugo de métodos geoestatísticos contribui para mostrar que se manejo adotado, influência a variabilidade espacial dos atributos do solo (SILVA et al., 2008).

Objetivou com este trabalho estudar a variabilidade espacial da porosidade total, da umidade gravimétrica do solo e da resistência do solo à penetração de um Argissolo Amarelo em uma área de pastejo rotacionado de ovinos.

\section{Material e Métodos}

O experimento foi conduzido numa área experimental no Núcleo de Ensino e Estudos em Forragicultura da Universidade Federal do Ceará, Campus do Pici, Fortaleza - CE. A área experimental situa-se na zona litorânea a 15,49 $\mathrm{m}$ de altitude, 3043'02" latitude Sul e 38³2'35" longitude Oeste localizada numa região de clima Aw' segundo a classificação de Köppen que se caracteriza por possuir um clima chuvoso, com precipitações de outono a verão e temperatura média maior de $18^{\circ} \mathrm{C}$ durante o ano inteiro. $\mathrm{O}$ solo estudado foi classificado como Argissolo Amarelo de textura arenosa segundo a classificação da Embrapa (2006). A área experimental era cultivada com Brachiaria decumbens e capim tifton, a qual vem sendo manejada sob lotação rotativa com ovinos e caprinos por aproximadamente 10 anos. As características físicas e propriedades químicas do solo da área de estudo encontram-se na Tabela 1 e foram obtidas conforme metodologia adotada pela Embrapa (1997).

Tabela 1. Características físicas e propriedades químicas do solo da área de estudo.

\begin{tabular}{ccccccccccc}
\hline $\begin{array}{c}\text { Densidade } \\
\text { do solo } \\
\left(\mathrm{g} . \mathrm{m}^{-3}\right)\end{array}$ & $\begin{array}{c}\text { Umidade } \\
\text { gravimétrica } \\
\left(\mathrm{g} .100 \mathrm{~g}^{-1}\right)\end{array}$ & $\mathrm{pH}$ & $\mathrm{V}$ & $\mathrm{P}$ & $\mathrm{MO}$ & $\begin{array}{c}\text { Areia } \\
\text { Grossa }\end{array}$ & $\begin{array}{c}\text { Areia } \\
\text { Fina }\end{array}$ & Silte & Argila & $\begin{array}{c}\text { Classificação } \\
\text { Textural }\end{array}$ \\
\hline 1,45 & 4,96 & 6,6 & 83 & 65 & 13,14 & 541 & 314 & 75 & 70 & Areia franca \\
\hline
\end{tabular}

pH em $\mathrm{H}_{2} \mathrm{O} ; \mathrm{V}$ em \%; P em Mg.kg ${ }^{-1}$; MO em g.kg ${ }^{-1}$; Areia, Silte e Argila em g.kg-1.

Fonte: Elaboração dos autores.

As amostras de solo foram retiradas nas camadas de $0-0,1 ; 0,1-0,2$ e $0,2-0,3 m$. Foi delimitado dentro de uma área de 1,28ha, uma malha onde foram demarcados 13 pontos georreferenciados para as coletas das amostras de solo deformadas e indeformadas e a resistência do solo à penetração, respeitando o espaçamento mínimo de 30m entre pontos. A porosidade total foi obtida através de amostras indeformadas de solo utilizandose amostrador de Uhland e anéis volumétricos com capacidade de $54 \mathrm{~m}^{3}$; a umidade do solo foi determinada pelo método gravimétrico realizado através da coleta de amostras deformadas, ambas, utilizando metodologia proposta pela Embrapa (1997) e a resistência do solo à penetração foi determinada, em cada ponto da malha, utilizando um penetrômetro eletrônico georreferenciado modelo PNT 2000 da DGL Automação®, com ponteira cônica de aço inoxidável, fixada na extremidade inferior da haste de penetração, com área basal de $130 \mathrm{~mm}^{2}$ conforme norma da Asae (2009) e a haste inserida ao solo até a camada de $0,3 \mathrm{~m}$.

A variabilidade do solo foi inicialmente avaliada através da análise descritiva dos dados, calculandose a média, mediana, variância, coeficiente de variação, coeficiente de assimetria e coeficiente de curtose, também foi realizada as analises de normalidade pelo teste de Shapiro-Wilks. Tal análise foi realizada através do programa SAS (SCHLOTZHAVER; LITTELL, 1997). A avaliação 
da variabilidade dos atributos, medida pelo coeficiente de variação (CV) foi baseada nos limites propostos por Warrick e Nielsen (1980), para classificação de atributos do solo, que consideram: variabilidade baixa $(\mathrm{CV}<12 \%)$; média $(12 \%<$ $\mathrm{CV}<60 \%)$ e alta $(\mathrm{CV}>60 \%)$. Para verificar a dependência espacial das variáveis e interpolar os dados foi empregada a análise geoestatística (VIEIRA, 2000). Foram construídos variogramas partindo das pressuposições de estacionaridade da hipótese intrínseca e do cálculo da semivariância estimada na equação 1 .

$$
\gamma^{*}(\mathrm{~h})=\frac{1}{2 N(\mathrm{~h})} \sum_{i=1}^{N(h)}\left[\mathrm{Z}\left(\mathrm{x}_{\mathrm{i}}\right)-\mathrm{Z}\left(\mathrm{x}_{\mathrm{i}}+\mathrm{h}\right)\right]^{2}
$$

em que $N(h)$ é o número de pares experimentais de observações $Z(x i)$ e $Z(x i+h)$ separados por uma distância h. O variograma é representado pelo gráfico $\gamma^{*}(\mathrm{~h})$ versUg $\mathrm{h}$.

Para a determinação do índice de dependência espacial (IDE), que é a proporção em porcentagem do efeito pepita $\left(\mathrm{C}_{\mathrm{o}}\right)$ em relação ao patamar $\left(\mathrm{C}_{\mathrm{o}}+\right.$ C), foi utilizado a equação $\left(\mathrm{C}_{\mathrm{o}} / \mathrm{C}_{\mathrm{o}}+\mathrm{C}\right)$ proposta por Cambardella et al. (1994) que considera dependência forte $<25 \%$; dependência moderada de 25 a $75 \%$; dependência fraca $>75 \%$. As análises geoestatísticas foram realizadas utilizando o programa GS+ (GAMMA DESIGN SOFTWARE, 2000) e a interpolação dos dados realizadas pelo método de krigagem para a confecção dos mapas através do programa computacional Surfer 9 (GOLDEN SOFTWARE, 2010).

\section{Resultados e Discussão}

A Tabela 2 apresenta os resultados da estatística descritiva para a porosidade total (PT), umidade gravimétrica do solo $(\mathrm{Ug})$ e Resistência do solo à penetração (RP) nas camadas de 0-0,1, 0,1-0,2 e $0,2-0,3 \mathrm{~m}$. Os coeficientes de assimetria e curtose indicam se a distribuição dos dados de uma variável está próxima à distribuição normal (coeficientes iguais a zero). A condição de normalidade não é um pré-requisito fundamental para o emprego da análise geoestatística, o importante é que a distribuição não apresente grandes fugas nas extremidades da curva, que poderiam comprometer a análise (GONÇALVES; FOLEGATTI; MATA, 2001). Segundo Webster e Oliver (2007), um coeficiente de assimetria dos dados entre os valores de 0 e 0,5 não indica a necessidade de transformação, um coeficiente entre 0,5 e 1,0 a transformação em raiz quadrada é a mais recomendável e um coeficiente maior que 1,0 , se faz necessário a transformação logarítmica dos dados. As variáveis não apresentaram altos valores para os coeficientes de assimetria e curtose, a distribuição dos dados foi considerada próxima à distribuição normal e a análise geoestatística foi realizada. A PT apresentou uma distribuição com assimetria negativa em todas as camadas analisadas, nesses casos, observou-se que a mediana é maior que a média, mostrando tendência para a concentração de valores maiores que essa. Os coeficientes de curtose apresentaram uma variação entre comportamentos leptocúrtica com uma maior concentração dos dados entorno torno da média e platicúrtica com um maior nível de achatamento da distribuição. Com exceção da PT, na camada de $0-0,1 \mathrm{~m}$, e da RP em todas as camadas estudadas, os demais atributos apresentaram distribuição normal pelo teste Shapiro-Wilks (W).

Segundo Carvalho, Soratto e Freddi (2002), o CV pode ser usado para comparar e avaliar a variabilidade entre duas variáveis. Assim na Tabela 1 podemos observar que a PT apresentou um CV baixo nas camadas de 0-0,1; 0,1-0,2 e 0,2 a $0,3 \mathrm{~m}$ indicando uma pequena variação dos dados em relação a média mostrando uma distribuição homogênea. Já a Ug em todas as camadas e a RP nas camadas de 0-0,1 e 0,1-0,2m apresentaram um CV médio. A RP na camada de 0,2-0,3m apresentou um CV alto, em relação às demais variáveis, indicando uma alta variação dos dados em relação à média mostrando uma distribuição com alta heterogeneidade nos dados. 
Tabela 2. Análise descritiva dos atributos do solo: Porosidade Total (PT), Umidade gravimétrica do solo (Ug) e Resistência do solo à penetração (RP) nas camadas de 0-0,1, 0,1-0,2 e 0,2-0,3m.

\begin{tabular}{ccccccccc}
\hline Variáveis & Média & Mediana & Desvio Padrão & Variância & CV & Assimetria & Curtose & W $^{*}$ \\
\hline \multicolumn{7}{c}{ Profundidade $0-0,1 \mathrm{~m}$} \\
\hline PT & 37,40 & 38,41 & 2,71 & 7,34 & 7 & $-1,0$ & 0,21 & Não \\
Ug & 6,43 & 6,30 & 2,12 & 4,49 & 33 & 0,80 & 0,86 & Sim \\
RP & 0,79 & 0,69 & 0,38 & 0,14 & 48 & 1,28 & 1,49 & Não \\
\hline \multicolumn{7}{c}{ Profundidade $0,1-0,2 \mathrm{~m}$} \\
\hline PT & 35,74 & 36,41 & 4,20 & 17,60 & 12 & $-0,83$ & $-0,30$ & Sim \\
Ug & 7,85 & 7,10 & 3,09 & 9,57 & 44 & 0,08 & 0,03 & Sim \\
RP & 1,74 & 1,41 & 1,01 & 1,01 & 58 & 2,15 & 2,12 & Não \\
\hline \multicolumn{7}{c}{ Profundidade $0,2-0,3 \mathrm{~m}$} \\
\hline PT & 35,40 & 35,79 & 3,64 & 13,28 & 10 & $-0,94$ & 0,38 & Sim \\
Ug & 7,79 & 7,14 & 3,55 & 12,60 & 46 & 0,46 & $-0,91$ & Sim \\
RP & 2,55 & 2,00 & 1,60 & 2,55 & 63 & 1,99 & 2,93 & Não \\
\hline
\end{tabular}

PT: Porosidade total (\%);Ug: Umidade do solo (g/g ); RP: Resistencia a penetração (MPa);

* teste de normalidade a $5 \%$ de probabilidade.

Fonte: Elaboração dos autores.

A Tabela 3 apresenta os modelos e parâmetros estimados aos semiovariogramas experimentais ajustados para os atributos físicos do solo estudado. É possível observar que através da análise dos semivariogramas foi constatado que todas as variáveis estudadas apresentaram um IDE fraco. O alcance é um importante parâmetro no estudo do semivariograma, pois representa a distância na qual não há correlação espacial entre os pontos de uma mesma variável. $\mathrm{O}$ alcance para $\mathrm{RP}$ apresentou maior valor (de $310,9 \mathrm{~m}$ ) na camada de $0,2-0,3 \mathrm{~m}$ e a Ug o menor valor $(39,3 \mathrm{~m})$ na camada de $0,2-$ $0,3 \mathrm{~m}$. A Ug nas camadas de $0-0,1$ e $0,2-0,3 \mathrm{~m}$ e a RP na camada de 0-0,1m apresentaram EPP, ou seja, não foi possível ajustar um modelo teórico aos semivariogramas experimentais uma vez que isso mostra que a distribuição espacial da Ug e da RP nesta área é aleatória e as amostras com a distância de $30 \mathrm{~m}$ são independentes. Segundo Cambardella et al. (1994), o efeito pepita constitui-se numa medida importante do semivariograma e indica a variabilidade não explicada, que pode ser devida a erros de medida e microvariações não detectadas, considerando a distância de amostragem utilizada.

A Figura 1 mostra os mapas de krigagem para a PT do solo nas camadas avaliadas com dependência espacial fraca. Nota-se que a PT é nos locais onde a RP exerce uma maior influência sobre o solo. Esta operação resulta na expulsão de ar dos poros, causando um rearranjamento das partículas, tornando o solo mais denso e conseqüente redução da porosidade (DIAS JÚNIOR; PIERCE, 1996). Ainda, de acordo com Mercante, Uribe-Opazo e Souza (2003) e Secco et al. (2005), a porosidade total tem apresentado grande relação com a compactação e a resistência à penetração do solo, as quais tendem a aumentar com a redução do espaço poroso. 
Tabela 3. Modelos e parâmetros estimados aos semiovariogramas experimentais: Porosidade Total (PT), Umidade gravimétrica do solo (Ug) e Resistência do solo à penetração $(\mathrm{RP})$ nas camadas de $0-0,1 ; 0,1-0,2$ e 0,2 a 0,3m.

\begin{tabular}{|c|c|c|c|c|c|c|}
\hline Atributos & Modelo & $\mathrm{Co}$ & $\mathrm{Co}+\mathrm{C}$ & A & IDE & $\mathrm{R}^{2}$ \\
\hline \multicolumn{7}{|c|}{ Camada $0-0,1 \mathrm{~m}$} \\
\hline PT & Esférico & 0,93 & 10,86 & 124,3 & 91 & 98 \\
\hline $\mathrm{Ug}$ & EPP & -- & -- & -- & -- & -- \\
\hline $\mathrm{RP}$ & EPP & -- & -- & -- & -- & -- \\
\hline \multicolumn{7}{|c|}{ Camada $0,1-0,2 \mathrm{~m}$} \\
\hline PT & Exponencial & 0,01 & 21,01 & 67,2 & 99 & 98 \\
\hline $\mathrm{Ug}$ & Exponencial & 1,16 & 11,07 & 39,3 & 90 & 26 \\
\hline $\mathrm{RP}$ & Esférico & 0,07 & 0,79 & 310,1 & 90 & 81 \\
\hline \multicolumn{7}{|c|}{ Camada $0,2-0,3 \mathrm{~m}$} \\
\hline PT & Esférico & 0,01 & 10,47 & 57,9 & 99 & 64 \\
\hline $\mathrm{Ug}$ & EPP & -- & -- & -- & -- & -- \\
\hline $\mathrm{RP}$ & Esférico & 0,08 & 0,81 & 310,9 & 90 & 84 \\
\hline
\end{tabular}

PT: Porosidade total (\%);Ug: umidade gravimétrica do solo (g ); RP:Resistencia do solo à penetração (MPa); $\mathrm{C}_{\mathrm{o}}$ : efeito pepita; $\mathrm{C}_{0}+\mathrm{C}_{1}$ : patamar; A: alcance da dependência espacial (m); IDE: índice de dependência espacial e $\mathrm{R}^{2}$ : coeficiente de determinação múltipla do ajuste.

Fonte: Elaboração dos autores.

Figura 1. Mapas de krigagem da porosidade total (PT) do solo nas camadas de 0-0,1, 0,1-0,2 e 0,2-0,3m.
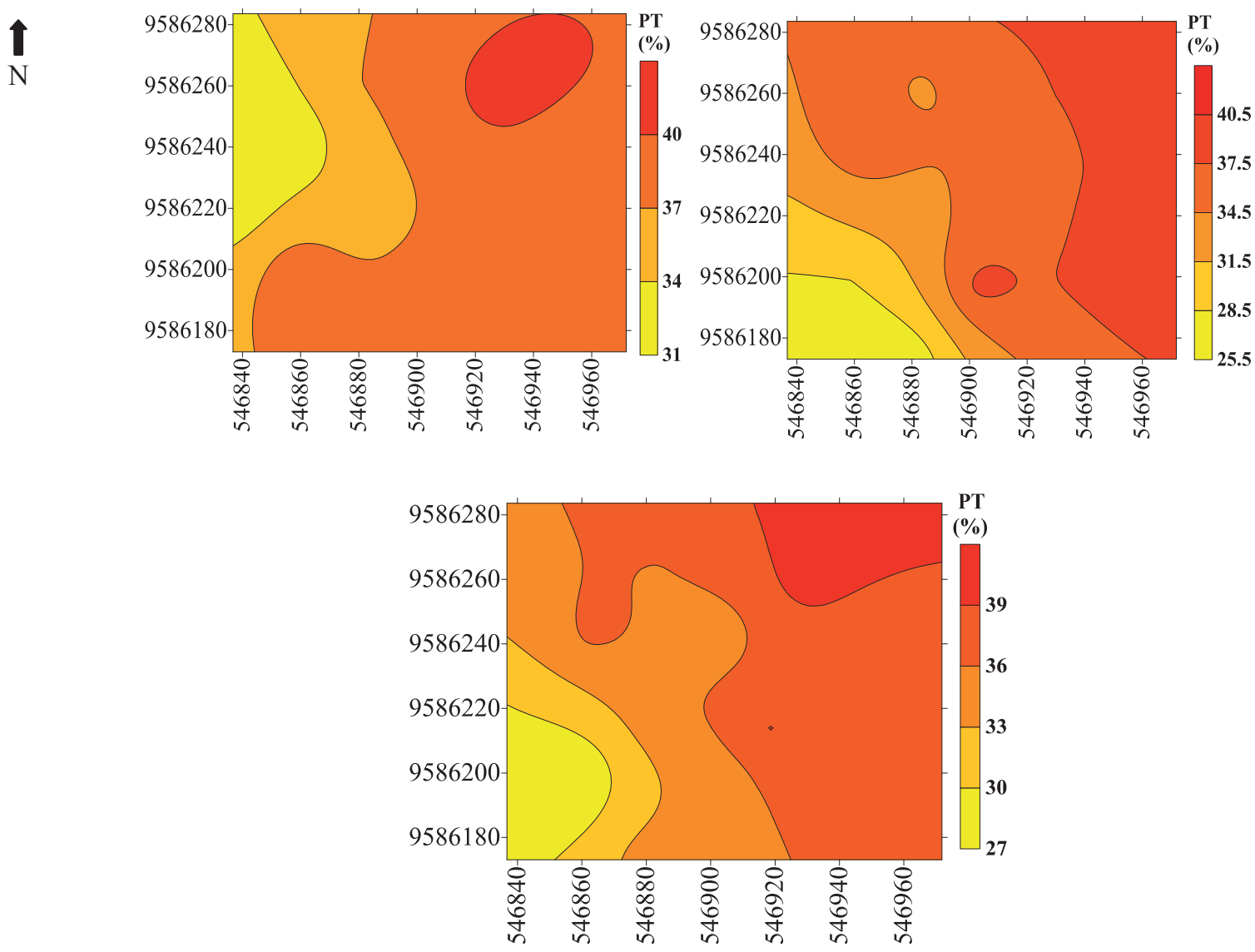

Fonte: Elaborado pelos autores. 
$\mathrm{Na}$ Figura 2 temos os mapas da krigagem para a resistência dos solo à penetração nas camadas de 0,1 0,2 e $0,2-0,3 \mathrm{~m}$, uma vez que na camada de $0-0,1 \mathrm{~m}$ a RP apresentou EPP. Observa-se que a RP variou de 0,6 MPa a 5,0 MPa, sendo que, a camada de 0,1$0,2 \mathrm{~m}$ apresentou uma média de $1,74 \mathrm{MPa}$ enquanto que na camada de 0,2-0,3m a média foi de 2,55 $\mathrm{MPa}$. Valores entre 2 e 2,5 MPa têm sido indicados como os limites críticos de resistência do solo à penetração para a maioria dos vegetais (SILVEIRA et al., 2010). Assumindo o valor de 2,5 MPa como o limite crítico ao crescimento das raízes (CANARACHE, 1990; CAMARGO; ALLEONI, 1997). No entanto, deve- se considerar que a resistência do solo à penetração possui relação potencial com a umidade do solo (BUSSCHER et al., 1997), o que pode explicar os valores encontrados para a amplitude e o coeficiente de variação, tendo em vista que pequenas alterações no conteúdo de água determinam grandes variações na grandeza dependente, ou seja, na resistência do solo à penetração. Nesse solo, esse comportamento decorre, possivelmente, da influência da textura do solo, cuja predominância da fração areia nas camadas avaliadas resulta em rápida permeabilidade e consequente variação no conteúdo de água no solo, transferindo essa característica para a resistência do solo à penetração.

Figura 2. Mapas de krigagem da resistência do solo à penetração nas camadas de 0,1-0,2 e 0,2-0,3m.
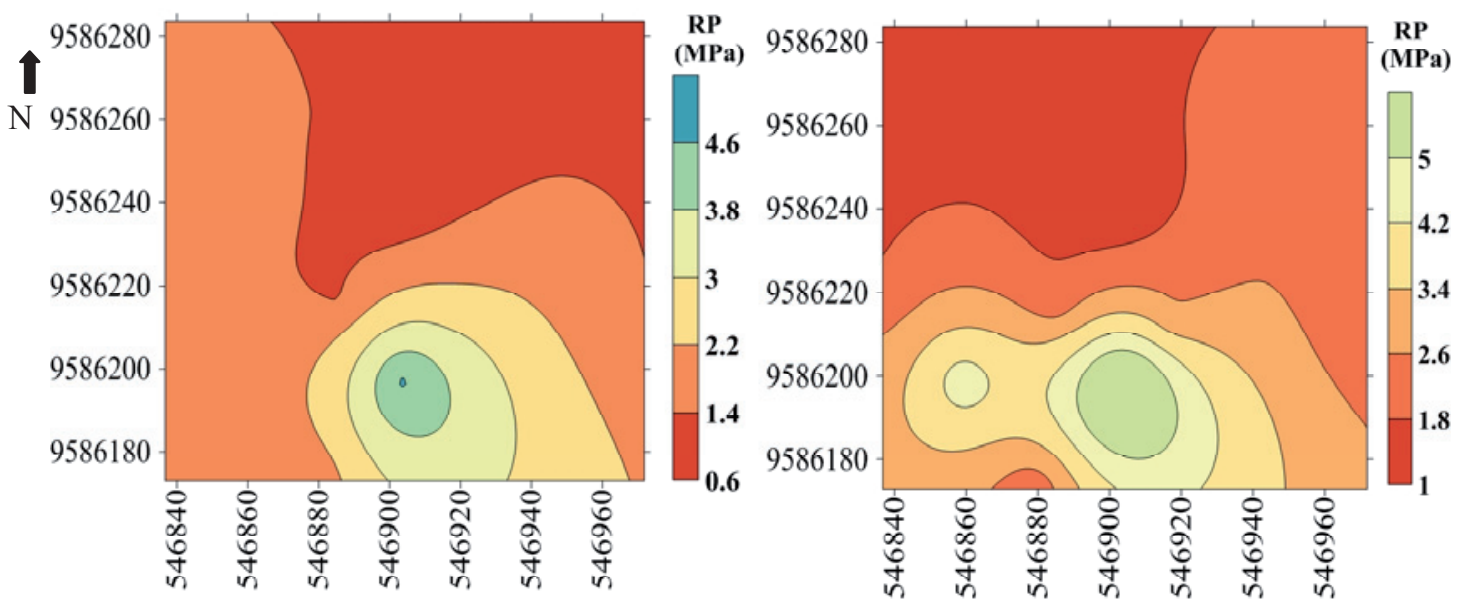

Fonte: Elaboração dos autores.

$\mathrm{Na}$ Figura 3 temos o mapa da Umidade gravimétrica do solo na camada $0,1-0,2 \mathrm{~m}$ onde mostra que a Ug apresentou uma relação inversa com a RP quando comparadas na mesma camada. Pelo mapa é possível observar que a medida que a Ug aumenta a RP diminui e isso ocorreu em todos os pontos estudados. Silveira et al. (2010) encontraram uma correlação negativa da Ug com a RP em um Argissolo Amarelo. Segundo Pedrotti, Pauletti e Crestana (2001), a manutenção de valores elevados de água na camada superficial do solo, principalmente, na presença contínua de cobertura vegetal em tratamentos sob plantio direto pode contribuir para a obtenção de menores valores de resistência do solo à penetração. Ela está diretamente correlacionada com vários atributos $\mathrm{e}$ condição do solo, como textura, densidade, matéria orgânica e, principalmente, a umidade no momento da determinação (STOLF; FERNANDES; VILANI NETO, 1983; BUSSCHER et al., 1997). Também pode ser influenciada pela coesão (GIAROLA et al., 2001). A umidade altera a coesão entre as partículas 
do solo (BELTRAME; GONDIN; TAYLOR, 1981). Assim, quando o solo está seco ou apresenta baixo conteúdo de água, suas partículas apresentam-se mais próximas e difíceis de serem separadas por qualquer força externa. Essa realidade é acentuada pela rapidez com que esses solos perdem água, tendo em vista sua elevada permeabilidade e textura franco-arenosa, pouco eficiente na retenção de água (SILVEIRA, 2009). É válido lembrar que a quantidade de água existente no solo está intimamente ligada aos valores de resistência do solo à penetração, e qualquer alteração neste último fator pode modificar tais valores (BOTTEGA et al., 2011).

Figura 3. Mapas de krigagem da umidade gravimétrica do solo na camada 0,1-0,2m.

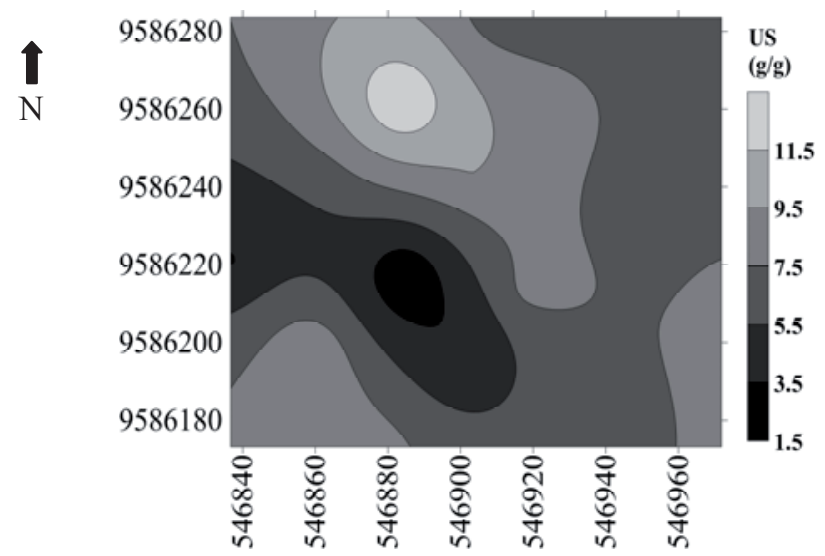

Fonte: Elaboração dos autores.

\section{Conclusão}

As variáveis PT, RP e UG apresentaram um índice de dependência espacial fraco em todas as camadas avaliadas.

A RP aumenta à medida que a PT e a UG exerce uma menor influência sobre o solo.

\section{Referências}

AMERICAN SOCIETY OF AGRICULTURAL ENGINEERS - ASAE. Soil cone penetrometer: ASAE S 313.9. ASAE STANDARDS 2009, St. Joseph, 2009.

BELTRAME, L. F. S.; GONDIN, L. A. P.; TAYLOR, J. C. Estrutura e compactação na permeabilidade de solos do Rio Grande do Sul. Revista Brasileira de Ciência do Solo, Viçosa, MG, v. 5, n. 1, p. 145-149, 1981.

BOTTEGA, E. L.; BOTTEGA, S. P.; SILVA, S. A.; QUEIROZ, D. M.; SOUZA, C. M. A.; RAFULL, L. Z. L. Variabilidade espacial da resistência do solo à penetração em um Latossolo Vermelho distroférrico. Revista Brasileira de Ciências Agrárias, Recife, v. 6, n. 2, p. 331-336, 2011.

BUSSCHER, W. J.; BAUER, P. J.; CAMP, C. R.; SOJKA, R. E. Correction of cone index for soil water content differences in a coastal plain soil. Soil Tillage Research, Amsterdam, v. 43, n. 43, p. 205-217, 1997.

CAMARGO, O. A.; ALLEONI, L. R. F. Compactação do solo e o desenvolvimento de plantas. Piracicaba: Escola Superior de Agricultura Luiz de Queiroz, 1997. 132 p.

CAMBARDELLA, C. A.; MOORMAN, T. B.; NOVAK, J. M.; PARKIN, T. B.; KARLEN, D. L.; TURCO, R. F.; KONOPKA, A. E. Field-scale variability of soil properties in central Iowa soils. Soil Science Society Amsterdam Journal, Madison, v. 58, n. 5, p. 1501-1511, 1994.

CANARACHE, A. PENETR - a generalized semiempirical model estimating soil resistance to penetration. Soil Tillage Research, Netherlands, v. 16, n. 1-2, p. 5170, 1990. 
CARVALHO, A. J. A.; SOUZA, E. H.; MARQUES, C. T. S.; GAMA, E. V. S.; NACIF, P. G. S. Caracterização física dos solos dos quintais agroflorestais e cultivos monotípicos na região de Amargosa, Bahia. Revista Brasileira de Agroecologia, Bahia, v. 2, n. 2, p. 941-944, 2007.

CARVALHO, M. P.; SORATTO, R. P.; FREDDI, O. $\mathrm{S}$. Variabilidade espacial de atributos físicos em um Latossolo Vermelho distrófico sob preparo convencional em Selvíria, estado de Mato Grosso do Sul. Acta Scientiarum, Maringá, v. 24, n. 5, p. 1353-1361, 2002.

CUNHA, J. P. A. R.; VIEIRA, L. B.; MAGALHÃES, A. C. Resistência mecânica do solo à penetração sob diferentes densidades e teores de água. Revista Engenharia na Agricultura, Viçosa, v. 10, n. 1-4, p. 1-7, 2002.

DIAS JÚNIOR, M. S.; PIERCE, F. J. Revisão de literatura: o processo de compactação do solo e sua modelagem. Revista Brasileira de Ciência do Solo, Viçosa, MG, v. 20, n. 1, p. 175-182, 1996.

EMPRESA BRASILEIRA DE PESQUISA AGROPECUÁRIA - EMBRAPA. Centro Nacional de Pesquisa de Solos. Sistema brasileiro de classificação de solos. 2. ed. rev. atual. Brasília, DF: Embrapa Produção da Informação. Rio de Janeiro: Embrapa Solos, 2006. $306 \mathrm{p}$.

Serviço Nacional de Levantamento e Conservação de Solos. Manual de métodos de análise do solo. 2. ed. Rio de Janeiro: Ministério da Agricultura e Abastecimento, 1997. 212 p.

EPSTEIN, E.; BLOOM, A. Nutrição mineral de plantas: princípios e perspectivas. 2. ed. Londrina: Planta, 2006. $403 \mathrm{p}$.

FUENTES, R. L.; RICHART, A.; TAVARES FILHO, J.; GUIMARÃES, M. F.; FERREIRA, R. R. M. Evolução de propriedades físicas do solo em função dos diferentes sistemas de preparo em culturas anuais. Semina: Ciências Agrárias, Londrina, v. 27, n. 2, p. 205-220, 2006.

GAMMA DESIGN SOFTWARE. GS+ for windows. Demonstration version. Michigan: Gamma Design Software, 2000.

GIAROLA, N. F. B.; SILVA, A. P.; TORMENA, C.; SOUZA, L. S.; RIBEIRO, L. P. Similaridades entre o caráter coeso dos solos e o comportamento Hardsetting: estudo de caso. Revista Brasileira de Ciência do Solo, Viçosa, v. 25, n. 1, p. 239-247, 2001.

GOLDEN SOFTWARE. Surfer for windows version 9.0. Colorado: Golden, 2010.
GONÇALVES, A. C. A.; FOLEGATTI, M. V.; MATA, J. D. V. Análises exploratória e geoestatística da variabilidade de propriedades físicas de um Argissolo Vermelho. Acta Scientiarum, Maringá, v. 23, n. 5, p. 1149-1157, 2001.

HU, W.; SHAO, M. A.; WANG, Q. J.; REICHARDT, K. Soil water content temporal spatial variability of the surface layer of a Loess Plateau hillside in China. Scientia Agrícola, Piracicaba, v. 65, n. 3, p. 277-289, 2008.

IMHOFF, S.; SILVA, A. P.; TORMENA, C. A. Aplicações da curva de resistência no controle da qualidade física de um solo sob pastagem. Pesquisa Agropecuária Brasileira, Brasília, v. 35, n. 7, p. 1403-1500, 2000.

KIEHL, E. J. Manual de edafologia: relações solo-planta. Piracicaba: Agronômica Ceres, 1979. 264 p.

MERCANTE, E.; URIBE-OPAZO, M. A.; SOUZA, E. G. Variabilidade espacial e temporal da resistência mecânica do solo à penetração em áreas com e sem manejo químico localizado. Revista Brasileira de Ciência do Solo, Viçosa, MG, v. 27, n. 6, p. 1149-1159, 2003.

OVALLES, F.; REY, J. Variabilidad interna de unidades de fertilidad en suelos de la depresión del Lago de Valencia. Agronomia Tropical, Madrid, v. 44, n. 1, p. 4165, 1994.

PEDROTTI, A.; PAUleTtO, E. A.; CRESTANA, S. Resistência mecânica à penetração de um Planossolo submetido a diferentes sistemas de cultivo. Revista Brasileira de Ciência do Solo, Viçosa, MG, v. 25, n. 3, p. 521-529, 2001.

SCHLOTZHAVER, S. D.; LITTELL, R. C. SAS: system for elementary statistical analysis. 2. ed. Cory: SAS, 1997.

SECCO, D.; ROS, C. O. D.; SECCO, J. K.; FIORIN, J. E. Atributos físicos e produtividades de culturas em um Latossolo Vermelho argiloso sob diferentes sistemas de manejo. Revista Brasileira de Ciência do Solo, Viçosa, MG, v. 29, n. 3, p. 407-414, 2005.

SILVA, J. M.; LIMA, J. S. S.; PIRES, F. R.; ASSIS, R. L. Variabilidade espacial dos atributos físicos em um Latossolo sob plantio direto e preparo convencional no cultivo da soja no cerrado. Revista Ciências Agrárias, Belém, v. 50, n. 50, p. 167-180, 2008.

SILVA, V. R.; REICHERT, J. M.; REINERT, D. J. Variabilidade espacial da resistência do solo à penetração em plantio direto. Ciência Rural, Santa Maria, v. 34, n. 2 , p. 399-406, 2004. 
SILVA, V. R.; REINERT, D. J.; REICHERT, J. M. Suscetibilidade à compactação de um Latossolo Vermelho-Escuro e de um Podzólico Vermelho-Amarelo. Revista Brasileira de Ciência do Solo, Viçosa, MG, v. 24, n. 2, p. 239-249, 2000.

SILVEIRA, D. C.; MELO FILHO, J. F.; SACRAMENTO, J. A. A. S.; SILVEIRA, E. C. P. Relação umidade versus resistência do solo à penetração para um Argissolo Amarelo distrocoeso no Recôncavo da Bahia. Revista Brasileira Ciência do Solo, Viçosa, MG, v. 34, n. 3, p. 659-667, 2010.

SILVEIRA, D.C. Diagnóstico da qualidade do solo em um pomar de citros cultivado com plantas de cobertura nas condições ambientais do Recôncavo da Bahia. 2009. Tese (Mestrado em Ciências Agrárias, Ambientais e Biológicas) - Universidade Federal do Recôncavo da Bahia, Cruz das Almas.

SOUZA, Z. M.; SILVA, M. L. S.; GUIMARÃES, D. T. S.; CARVALHO, M. P.; PEREIRA, G. T. Variabilidade espacial de atributos físicos em um Latossolo Vermelho distrófico sob semeadura direta em Selvíria (MS). Revista Brasileira de Ciência do Solo, Viçosa, MG, v. 25, n. 3, p. 699-707, 2001.
STOLF, R.; FERNANDES, J.; URLANI NETO, V. L. Recomendação para o uso do penetrômetro de impacto - modelo IAA/Planalsucar - Stolf. São Paulo: MIC/IAA/ PNMCA-Planalsucar, 1983. 8 p. (Boletim, 1).

TIMM, L. C.; PIRES, L. F.; ROVERATTI, R.; ARTHUR, R. C. J.; REICHARDT, K.; OLIVEIRA, J. C. M.; BACCHI, O. O. S. Field spatial and temporal patterns of soil water content and bulk density changes. Scientia Agrícola, Piracicaba, v. 63, n. 1, p. 55-64, 2006.

TRANGMAR, B. B.; YOST, R. S.; WADE, M. K.; UEHARA, G. Applications of geostatistics to spatial studies of soil properties. Advances in Agronomy, San Diego, v. 38, n. 1, p. 45-94, 1985.

VIEIRA, S. R. Geoestatística em estudos de variabilidade espacial do solo. In: NOVAIS, R. F.; ALVARES, V. V. H.; SCHAEFFER, C. E. G. R. (Ed.). Tópicos em ciência do solo. Viçosa: Sociedade Brasileira de Ciência do Solo, 2000. p. 1-54.

WARRICK, A. W.; NIELSEN, D. R. Spatial variability of soil physical properties in the field. In: HILLEL, D. (Ed.). Applications of soil physics. New York: Academic, 1980. p. 319-344.

WEBSTER, R.; OLIVER, M. A. Geostatistics for environmental scientists. ${ }^{\text {td }}$ ed. Wiley: Chichester, 2007. $325 \mathrm{p}$. 